\title{
A study of fungal corneal ulcer
}

\author{
Meena $\mathbf{V}^{1}$, Sharma $\mathbf{U}^{2}$ \\ ${ }^{1}$ Dr. Vandana Meena, Medical Officer, Department of Ophthalmology, Gandhi Medical College, Bhopal, MP, India. \\ ${ }^{2}$ Dr. Unnati Sharma, Ophthalmolgy, GMC, Bhopal, MP, India.
}

Address for Correspondence: Dr. Vandana Meena, Medical Officer, Department of Ophthalmology, Gandhi Medical College, Bhopal, MP, India. Email: meena.vandana@gmail.com

\begin{abstract}
Background: Fungal keratitis is an inflammation of the cornea caused by fungi. This infection is difficult to treat and it can lead to severe visual impairment or blindness. It is worldwide in distribution, but is more common in the tropics and subtropical regions. Corneal ulcers are the second most-common cause of preventable blindness after cataract in tropical developing countries. Material \&Methods: A retrospective study included 30 cases of fungal corneal ulcer was carried out on indoor and outdoor cases of fungal corneal ulcer, in Department of ophthalmology, in technical collaboration with department of microbiology in tertiary care teaching hospital. A presumptive diagnosis was based on clinical features and history, diagnosis was confirmed by $\mathrm{KOH}$ preparation and culture. Results: The maximum incidence of ulcers was seen in age group of 41-60 years and majority of them were males. Most common predisposing factor was trauma. Most common clinical feature was slough followed by hypopyon, perforation, lowered intraocular tension, satellite lesions and vascularization respectively. Conclusion: The diagnosis of fungal keratitis is usually difficult. The clinical suspicion by ophthalmologist is unequivocally, key element in making diagnosis of fungal infection of cornea. A wide range of conventional and molecular techniques are currently available for laboratory diagnosis of fungal keratitis. Early diagnosis and appropriate treatment are essential to avoid blindness.
\end{abstract}

Keywords: Corneal, Fungal keratitis, Microbiology

\section{Introduction}

Fungal keratitis is primarily seen in tropical climates and is rare in temperate areas. Its incidence is between $6 \%-$ $20 \%$ of all microbial keratitis cases depending on the geographic location $[1,2]$. Traditionally, it is considered a disease of rural areas and is frequently caused by trauma with vegetative material. However, the major risk factor in developed countries is contact lens use at this time [3]. Its incidence has been reported to be increasing due to widespread use of contact lenses, especially bandage contact lenses, and topical steroid usage $[3,4]$ while tropical climates show a preponderance of filamentous fungi, temperate climates show higher percentages of yeast infections[5,6]. Ocular fungal infections, or ophthalmic mycoses, are being increasingly recognized as an important cause of morbidity and blindness; certain types of ophthalmic mycoses may even be life-threatening [7,8]. Keratitis (corneal infection) is the most frequent presentation [9],

Manuscript received: $22^{\text {nd }}$ Jan 2016

Reviewed: $01^{\text {st }}$ Feb 2016

Author Corrected: $10^{\text {th }} \mathrm{Feb} 2016$

Accepted for Publication: $20^{\text {th }}$ Feb 2016 but the orbit, lids, lacrimal apparatus, conjunctiva, sclera, and intraocular structures may also be involved. Fungi are opportunistic in the eye, since they rarely infect healthy, intact ocular tissues. Even the trivial trauma of a dust particle falling on the cornea may disrupt the integrity of the corneal epithelium, predisposing to mycotic keratitis. In compromised or immunosuppressed individual, serious sight-threatening and life-threatening infections such as rhinoorbitocerebralzygomycosis may supervene [8].

The potassium hydroxide $(\mathrm{KOH})$ wet mount and its modifications are widely used for the rapid detection of fungal hyphae in necrotic tissue samples from patients with infections of the orbit[10] and other ocular structures[11]. Several limitations have been reported when such mounts are used for corneal scrapes, including low sensitivity, frequent misinterpretation, presence of artifacts, and lack of detection ofCandida and other yeasts [10]. Moreover, if no dye or ink is added, the 
microscopist is looking for a usually colorless fungus against a colorless background; that is, there is no contrast to facilitate the detection of the fungal organisms.

This may explain why American ophthalmologists currently seem to prefer other techniques for detection of fungal elements in corneal scrapes. However, elsewhere, relatively good sensitivities have been reported in the diagnosis of culture-proven mycotickeratitis $[12,13,14,15.16]$.

A better understanding of pathogenetic mechanisms in ophthalmic mycoses is required. In particular, the possible role of fungal extracellular proteinases [17] and fungal morphogenesis [18] in ophthalmic mycoses requires clarification.

The role of nonspecific inflammatory mechanisms and specific immunological mechanisms in the pathogenesis of ophthalmic mycoses needs to be studied. A better understanding of these various pathogenetic mechanisms will permit the development of molecules and methods to neutralize these mechanisms and to augment antifungal therapy.

\section{.Material \& Methods}

A retrospective study fungal corneal ulcer was carried out on indoor and outdoor cases of fungal corneal ulcer in Department of ophthalmology, in technical collaboration with department of microbiology in tertiary care teaching hospital.

The study included 50 cases of fungal corneal ulcer who attended over a period of 1 year.

Inclusion criteria- Only cultures found positive for corneal fungal growth and fungal isolates were included in this study.

Exclusion criteria- There were no criteria for exclusion.

A standardized proforma was filled up for each patient with documentation of sociodemographic features, duration of symptoms, predisposing factors, history of trauma, associated ocular and systemic conditions, prior therapy received and all other clinical findings including visual acuity.

Visual acuity at the time of presentation was recorded. All the patients were examined by slit lamp biomicroscope by an ophthalmologist. After staining the ulcer with sodium fluroscin the size of the ulcer, stromal infiltrate and depth was measured using the variable slit on the slit lamp and recorded in millimeter.

The hypopyon if present was noted and measured similarly in millimeters. The ulcer margin, thinning of the floor, satellite lesions, any retained foreign body and pigmentation over the ulcer surface was recorded.

A diagram of each ulcer was drawn on the standardized form by performing frontal and cross sectional sketches. Associated ocular conditions like blepharitis, conjunctivitis, dacryocystitis, corneal anesthesia, dry eyes, lid abnormalities, lagophthalmos, past surgery in the cornea, use of contact lens and corticosteroids. Corneal scrapings were taken after instillation of $4 \%$ preservative free lignocaine by an ophthalmologist using no.15 Bard-Parker blade with strict asepsis under slitlamp biomicroscope.

The material collected from the leading edge and base of the ulcer was inoculated into solid/liquid culture media and two smears made onto two slides. One slidewas stained with Gram stain and the other with $10 \% \mathrm{KOH}$ preparation for direct microscopic examination.

Laboratory procedure: Aerobical incubations were done for all inoculated media. The inoculated Sabouraud's dextrose agar media were incubated at 270 $\mathrm{C}$ and after daily examination they were discarded after 3 weeks, if there was no growth.

The blood agar, chocolate agar, thioglycolate broth and brain-heart infusion broth after inoculation were incubated at $370 \mathrm{C}$, examined daily and if no growth was seen in 7 days were discarded.

When $\mathrm{KOH}$ wet mount preparations were positive for amoebic cysts, scrapings were inoculated in non-nutrient agar media overlaid with Escherichia coli. If, there was no growth in 3 weeks they were discarded.

All laboratory methods were performed following standard protocols $[19,20]$.

Microbial cultures were considered positive if there was growth of same organism in more than one solid phase media, and/or confluent growth at the site of inoculation on one solid medium and/or growth in one medium consistent with direct microscopic observations as in $\mathrm{KOH}$ preparation or Gram stain [19]. 


\section{Results}

Table No1: Age Incidence

\begin{tabular}{|c|c|c|c|}
\hline S.No. & Age GP In Years & Number & \% \\
\hline 1 & $0-10$ & 3 & 12 \\
\hline 2 & $11-20$ & 6 & 12 \\
\hline 3 & $21-30$ & 10 & 20 \\
\hline 4 & $31-40$ & 12 & 12 \\
\hline 5 & $41-50$ & 6 & 4 \\
\hline 6 & $51-60$ & 2 & 10 \\
\hline 7 & $61-70$ & 5 & $\mathbf{5 0}$ \\
\hline 8 & $71-80$ & Total & 6 \\
\hline
\end{tabular}

All age groups are affected but elderly age group 41-60 years (40\%) are most commonly affected.

Table No 2: Sex Incidences

\begin{tabular}{|c|c|c|}
\hline Sex & Number & \% \\
\hline Male & 36 & 72 \\
\hline Female & 14 & 28 \\
\hline
\end{tabular}

Males were predominantly affected. The male - female ratio was 2.70:1.

Table No 3: Incidence of Predisposing Factors

\begin{tabular}{|c|c|c|c|c|}
\hline Predisposing Factor & No. of ulcers & Percent & Contributing factors & Percent \\
\hline Trauma & 40 & 80 & 15 & 30 \\
\hline Foreign Body & 10 & 20 & 20 & 40 \\
\hline
\end{tabular}

Majority of patients had trauma to the eye as most common predisposing factor, $30 \%$ of these patients had one or more contributing factors. $33.3 \%$ pateints had foreign body into the eye as predisposing factor. $40 \%$ of these patients had one or more contributory factor.

These factors are entropion, chronic dacrocystitis, previous corneal opacity, use of antibiotic and or steroid prior to trauma or foreign body, diabetes mellitus, alcoholism or preceding illness.

Table No 4: Nature of Trauma

\begin{tabular}{|c|c|c|}
\hline Object & No. of pateints & Percent \\
\hline Vegetable matter & 27 & 54 \\
\hline Animal hair[cattle] & 4 & 4 \\
\hline Self inflicted[accidently] & 2 & 34 \\
\hline Foreign body & 17 & 8 \\
\hline
\end{tabular}


Table No 5: Clinical features and associated findings

\begin{tabular}{|c|c|c|}
\hline Findings & No. of Patients & Percent \\
\hline Slough & 50 & 800 \\
\hline Hypopyon & 42 & 78 \\
\hline Intraocular & & 14 \\
\hline Tension & 39 & 4 \\
\hline Normal & 7 & 60 \\
\hline Raised & 2 & 40 \\
\hline Lowered & 30 & 4 \\
\hline Satellite lesions & 20 & 68 \\
\hline Vascularization & 2 & 34 \\
\hline Perforation & & \\
\hline
\end{tabular}

Most common clinical feature was slogh followed by hypopyon, perforation, lowered intraocular tension, satellite lesions and vascularization respectively.

\section{Discussion}

Infectious keratitis remains an important cause of corneal ulcers in the developing countries. Studies have shown variable trends in risk factors, microbial profiles, and surgical outcomes of fungal keratitis [21,22]. In our study Majority of patients had trauma to the eye as most common predisposing factor, $30 \%$ of these patients had one or more contributing factors. $20 \%$ pateints had foreign body into the eye as predisposing factor. $40 \%$ of these patients had one or more contributory factor. These factors are entropion, chronic dacrocystitis, previous corneal opacity, use of antibiotic and or steroid prior to trauma or foreign body, diabetes mellitus, alcoholism or preceding illness which is similar to study done by Sharma K et al [23], They found Ocular trauma $68.3 \%$ was the most common predisposing factor followed by topical steroids (19.23\%) and diabetes mellitus (7.69\%). Katara RS et al[24] (trauma 44.45\%, diabetes mellitus $29.5 \%$, contact lens wearers $14.82 \%$ \& steroid $3.70 \%$ ) and Kumar et al[25] where ocular trauma constituted $78.5 \%$ of the cases.

This may be because most of the patients in the present study were involved in agriculture occupation where injury to the eye was very common. Another predisposing factor was use to topical steroids (14.6\%). This could to due to the easy availability of the steroid eye drops in our country.

Moreover, due to illiteracy, patients keep on using these eye drops continuously for longer periods, many times even without prescription.
In our study maximum case of the trauma was due to vegetable matter which is most common nature of trauma, followed by animal hair, foreign body, self inflicted [accidently], which is similar to Sharma A et al [23]. Trauma due to vegetable origin (75\%), sugarcane leaf and paddy leaf accounted for $50 \%$ and $16.6 \%$ of the cases respectively. This is in accordance to sharma $\mathrm{K}$ et al [23] (54.54\%) and Katara RS et al[24]] (62\%). Injury with sugarcane and paddy leaf predominates as they were the principal agricultural products in this region and majority of the farming community are engaged in these two crops. Sugarcane leaf because of its length can easily injure the eye during harvesting of crop.

In the present study, Most common clinical feature was slogh followed by hypopyon, perforation, lowered intraocular tension, satellite lesions, and vascularization respectively which is comparable with other studies the clinical features redness $(81.25 \%)$, blurred/diminished vision $(81.25 \%)$, pain $(68.7 \%)$, irregular feathery margins $(75 \%)$ was most commonly seen in fungal keratitis. Pain $(87.5 \%)$, redness $(87.5 \%)$, lacrimation (62.5\%), hypopyon $(37.5 \%)$ was most commonly seen in bacterial keratitis [23].

This was comparable with Ibrahim et al[26] (Red eye Bacterial 89.22\% fungal 87\%, Pain - Bacterial 90.32\% Fungal $87.55 \%$,Photophobia-Bacterial $67.74 \%$ Fungal $86.67 \%$,Poor vision -Bacterial 71.67\% Fungal-93.49\%, Hypopyon (Bacterial 36\% Fungal 16\%) and Thomas et al[27] (Serrated margins- fungal $79 \%$ bacterial $48 \%$, 
Hypopyon- fungal $48 \%$ bacterial $65 \%$, dry texturefungal $44 \%$ bacterial $28 \%$ ). Fungal aetiology is mostly presumed with hyphal pattern, serrated margins, raised slough, dry textured slough, and satellite lesions.

Joanne $\mathrm{W}$ et al[28] found a high prevalence of mixed bacterial-fungal corneal infections, representing 24 of 63 cases $(38 \%)$. This is significantly higher than the prevalence of bacterial-fungal infections reported in the Northeastern USA $(11 / 61,18 \%)$ [8]. This increased rate of polymicrobial infections may reflect the high number of patients with prior PKP in our series as these patients were likely more susceptible to superinfections. In contrast, bacterial aetiology is suspected when symptoms are more prominent.

It is marked by clinical features i.e.; flat, dry slough, margins well defined, hypopyon, keratic precipitates, flare or cells in the anterior chamber (AC), and deep lesions, but the practical experience in treating cases of fungal keratitis shows that the clinical features do not always correlate with the textbook description. Certain clinical characteristics of corneal ulcers may suggest a specific pathogen, but a reliable diagnosis cannot be made by clinical appearance alone and microbiological investigations should be performed.

\section{Conclusion}

Fungal keratitis is an avoidable vision-threatening disease that still represents a considerable proportion of the daily new cases that creates a huge burden on the resources of health services. The clinical presentations of bacterial and fungal corneal ulcers are often overlapping and thus confirmation by microbiological diagnosis is very essential in order to limit the ocular morbidity and prevent complications.

Future research in ophthalmic mycoses needs to focus on improvement in diagnostic techniques, development of new antifungal compounds and a better understanding of the pathogenesis of the conditions

\section{References}

1. Liesegang TJ, Forster RK. Spectrum of microbial keratitis in South Florida. Am J Ophthalmol. 1980 Jul;90(1):38-47.

2. Gopinathan U, Sharma S, Garg P, Rao GN. Review of epidemiological features, microbiological diagnosis and treatment outcome of microbial keratitis: experience of over a decade. Indian J Ophthalmol.2009;57:273-279.
3. Iyer SA, Tuli SS, Wagoner RC. Fungal keratitis: emerging trends and treatment outcomes. Eye Contact Lens. 2006 Dec;32(6):267-71.

4.GowerEW, KeayLJ, Oechsler RA, Iovieno A, Alfonso EC, Jones DB, Colby K, Tuli SS, Patel SR, Lee SM, Irvine J, Stulting RD, Mauger TF, Schein OD. Trends in fungal keratitis in the United States, 2001 to 2007. Ophthalmology. 2010 Dec;117(12):2263-7. doi: 10.1016/j.ophtha.2010.03.048. Epub 2010 Jun 29.

5. Gopinathan U, Garg P, Fernandes M, Sharma S, Athmanathan S, Rao GN. The epidemiological features and laboratory results of fungal keratitis: a 10year review at a referral eye care center in South India. Cornea. 2002 Aug;21(6):555-9.

6. Ritterband DC, Seedor JA, Shah MK, Koplin RS, McCormick SA. Fungal keratitis at the new york eye and ear infirmary. Cornea. 2006 Apr;25(3):264-7.

7. Levin LA, Avery R, Shore JW, Woog JJ, Baker AS. The spectrum of orbital aspergillosis: a clinicpathological review. Surv Ophthalmol. 1996 Sep-Oct; 41(2):142-54.

8. Yohai RA, Bullock JD, Aziz AA, Markert RJ. Survival factors in rhino-orbital-cerebral mucormycosis. Surv Ophthalmol. 1994 Jul-Aug;39(1):3-22.

9. Srinivasan R, Kanungo R, Goyal JL. Spectrum of oculomycosis in South India. Acta Ophthalmol (Copenh). 1991 Dec;69(6):744-9.

10. Rao NA. A laboratory approach to rapid diagnosis of ocular infections and prospects for the future. Am J Ophthalmol. 1989 Mar 15;107(3):283-91.

11. Rosa RH Jr, Miller D, Alfonso EC. The changing spectrum of fungal keratitis in south Florida. Ophthalmology. 1994 Jun;101(6):1005-13.

12. Gopinathan, U., P. Garg, M. Fernandes, S. Sharma, S. Athmanathan, and G. N. Rao. 2002. The epidemiological features and laboratory results of fungal keratitis. A 10-year review at a referral eye care center in South India. Cornea 21:555-559.

13. Marcus L, Vismer HF, van der Hoven HJ, Gove E, Meewes P. Mycotic keratitis caused by Curvularia brachyspora (Boedjin). A report of the first case. Mycopathologia. 1992 Jul;119(1):29-33. 
14. Sharma, S., M. Silverberg, P. Mehta, U. Gopinathan, V. Agrawal, and T. J. Naduvilath. 1998. Early diagnosis of mycotic keratitis: predictive value of potassium hydroxide preparation. Indian J. Ophthalmol. 46:3135 .

15. Wong TY, Ng TP, Fong KS, Tan DT. Risk factors and clinical outcomes between fungal and bacterial keratitis: a comparative study. CLAO J. 1997 Oct;23 (4):275-81.

16. Xie L, Dong X, Shi W. Treatment of fungal keratitis by penetrating keratoplasty. Br J Ophthalmol. $2001 \mathrm{Sep}$; 85(9):1070-4.

17. Zhu WS, Wojdyla K, Donlon K, Thomas PA, Eberle HI. Extracellular proteases of Aspergillus flavus. Fungal keratitis, proteases, and pathogenesis. Diagn Microbiol Infect Dis. 1990 Nov-Dec;13(6):491-7.

18. Thomas PA, Garrison RG, Jansen T. Intrahyphal hyphae in corneal tissue from a case of keratitis due to Lasiodiplodia theobromae. J Med Vet Mycol. 1991;29 (4): 263-7.

19.Srinivasan M, Gonzales CA, George C, Cevallos V, Mascarenhas JM, Asokan B, Wilkins J, Smolin G, Whitcher JP. Epidemiology and aetiological diagnosis of corneal ulceration in Madurai, south India. Br J Ophthalmol. 1997 Nov;81(11):965-71.

20. Sharma S,Athmanathan (2002). Diagnostic procedures in infectious keratitis. In; Nema H V, Nema $\mathrm{N}$, editors. Diagnostic procedures in Ophthalmology.New Delhi: Jaypee Brothers Medical Publishers; 232-253.
21. Tuli SS. Fungal keratitis. Clin Ophthalmol. 2011;5:275-9. doi: 10.2147/ OPTH. S10819.

22. Thomas PA. Current perspectives on ophthalmic mycoses. Clin Microbiol Rev. 2003 Oct;16(4):730-97.

23.Dr Sharma K, DrMehtaSA profile of corneal ulcers 2 years study from rural hospital, HaryanaIOSR Journal of Dental and Medical Sciences (IOSR-JDMS) e-ISSN: 2279-0853, p-ISSN: 2279-0861.Volume 13, Issue 11 Ver. VII (Nov. 2014), PP 94-97.www.iosrjournals.org

24.Katara RS, Patel ND, Sinha M. A Clinical Microbiological Study of Corneal Ulcer Patients at Western Gujarat, India.ActaMedicaIranica. 2013; 51(6):399-403.

25. Kumar A, Pandya S, Kavathia G, Antala S, Madan M, Javdekar T. Microbial keratitis in Gujarat, Western India: findings from 200 cases. Pan Afr Med J. 2011;10:48. Epub 2011 Nov 29.

26. Ibrahim MM, Vanini R, Ibrahim FM, Fioriti LS, Furlan EMR, Rovinzano LMA, De Castro RS, E Faria E Sousa SJ, Rocha EM. Epidemiologic aspects and clinical outcome of fungal keratitis in southeastern Brazil.E Journal Ophthalmol. 2009; 19(3):355-61.

27. Thomas PA, Leck AK, Myatt M. Characteristic clinical features as an aid to the diagnosis of suppurative keratitis caused by filamentous fungi. $\mathrm{Br} J$ Ophthalmol. 2005 Dec;89(12):1554-8.

28. Ho JW, Fernandez MM, Rebong RA, Carlson AN, Kim T, Afshari NA. Microbiological profiles of fungal keratitis: a 10 -year study at a tertiary referral center. J Ophthalmic Inflamm Infect. 2016 Dec;6(1):5. doi: 10.1186/s12348-016-0071-6. Epub 2016 Feb 20.

\section{How to cite this article?}

Meena V, Sharma U. A study of fungal corneal ulcer. Int J Med Res Rev 2016;4(3): 324-329. doi: 10.17511/ijmrr. 2016. i03.07. 